\title{
Evaluation of a multisensorial system for a rapid preliminary screening of the olive oil chemical compounds in an industrial process
}

\author{
Antonia Tamborrino, ${ }^{1}$ Alessandro Leone, ${ }^{1}$ Roberto Romaniello, ${ }^{2}$ Simone Grasso,${ }^{3}$ \\ Alessandro Zompanti, ${ }^{4}$ Maurizio Servili, ${ }^{5}$ Pablo Juliano, ${ }^{6}$ Marco Santonico ${ }^{3}$ \\ ${ }^{1}$ Department of Agricultural and Environmental Science, University of Bari Aldo Moro, Bari, Italy; ${ }^{2}$ Department of the \\ Science of Agriculture, Food and Environment, University of Foggia, Foggia, Italy; ${ }^{3}$ Department of Science and \\ Technology for Humans and the Environment, Unit of Electronics for Sensor Systems, Università Campus Bio-Medico, \\ Rome, Italy; ${ }^{4}$ Department of Engineering, Unit of Electronics for Sensor Systems, Università Campus Bio-Medico, \\ Rome, Italy; ${ }^{5}$ Department of Agricultural, Food and Environmental Science, University of Perugia, Perugia, Italy; \\ ${ }^{6}$ CSIRO Agriculture and Food, Werribee VIC, Australia
}

\begin{abstract}
In this study, a sensory system, named BIONOTE, based on gas and liquid analyses was used to analyse the headspace of olive oil samples obtained at the end of the extraction process for a preliminary screening of the volatile and phenolic compounds. Olive oil samples were obtained using different olive paste conditioning systems, including microwave and megasound machines at different processing time. The same olives batch was used for the entire test. BIONOTE showed the ability to discriminate between 64 virgin olive oils originated from different technologies or by using different process parameters, as demonstrated by the partial least square discriminant analysis (PLS-DA) models calculated. The percentage of correct classification in different conditions are in a range from $92.19 \%$ to $100 \%$. In addition, the research shown that the multisensorial system can provide a preliminary estimation of some volatile and phenolic compounds concentrations detected by laboratory analysis. Data analysis has been performed using multivariate data analysis techniques: PLS-DA cross validation via leave one out criterion. Future perspectives are to further develop BIONOTE in order to increase the number of detected chemical compounds and finally to include the mathematical models obtained in the BIONOTE microcontroller for a rapid chemical characterization of olive oil in the mill.
\end{abstract}

Correspondence: Antonia Tamborrino, Department of Agricultural and Environmental Science, University of Bari Aldo Moro, via Amendola 165/A, 70126 Bari, Italy.

E-mail: antonia.tamborrino@uniba.it

Key words: Virgin olive oil; microwave; ultrasound; chemical sensors; malaxation.

Received for publication: 16 September 2019.

Accepted for publication: 26 January 2020.

(C) Copyright: the Author(s), 2020

Licensee PAGEPress, Italy

Journal of Agricultural Engineering 2020; LI:1016

doi:10.4081/jae.2020.1016

This article is distributed under the terms of the Creative Commons Attribution Noncommercial License (by-nc 4.0) which permits any noncommercial use, distribution, and reproduction in any medium, provided the original author(s) and source are credited.

\section{Introduction}

Extra virgin olive oil (VOO) is an important food in the Mediterranean region highly appreciated by consumers for its healthful characteristics attributed to phenolic compounds and for the pleasantness of its aroma, characterized by various volatile compounds such as the carbonyl compounds, alcohols, esters and hydrocarbons (Flath et al., 1973; Servili et al., 2009).

Sensory descriptors of olive oil aroma can be classified into positive attributes, such as fruity, bitter and pungent, and defects. Top quality VOOs are characterized by having positive attributes and absence of negative ones.

Phenolic compounds, which are also responsible for its bitterness and pungency are, phenolic alcohols (like tyrosol and hydroxytyrosol) and secoiridoid derivatives such has oleacein, oleuropein aglycon and olecanthal. (Genovese et al., 2018; Servili et al., 2009). The main components responsible for the aroma are C6 and C5 substances, especially C6 linear unsaturated and saturated aldehydes and alcohols. They represent the most important fraction of the volatile compounds. These compounds are produced during mechanical extraction of the oil by the lipoxygenase (LOX) pathway using the polyunsaturated fatty acids as substrate. C6 and C5 concentrations are related to the activity of each involved enzyme (Salas and Sánchez, 1999; Angerosa et al., 2000; Angerosa et al., 2004).

The different shades of aroma of virgin olive oils are linked to the level and composition of the volatile and phenolic fraction.

Currently, the Panel Test method is the tool to measure olive oil quality and to determinate its positive attributes and defects. The Panel of expert, certified by the European Commission, operate according to the Regulation No. 2568/91. Although the sensory analysis plays an important role in VOO quality determination, it does not allow to characterize phenolic and volatile compounds.

Phenolic compounds can be analysed using various analytical laboratory methods that include the use of high performance liquid chromatography (HPLC) analysis, while the volatile compounds can be analysed using various analytical methods that include the use of solid phase micro extraction (SPME) technique and gas-chromatography coupled to a mass spectrometer (GCMS) (Cavalli et al., 2003; Angerosa et al., 2004).

In both cases although these methods of laboratory analysis are very efficient they are also very laborious, time consuming and expensive (Esposto et al., 2009).

As demonstrated in many scientific researches, the mechanical extraction process, in particular the technologies and process 
parameters adopted, strongly influence the olive oil volatile and phenolic compounds concentrations (Leone et al., 2013; Tamborrino et al., 2014; Leone et al., 2015a, 2015b, 2018).

During the oil extraction process, the ability to know the phenolic and volatile content of the oil currently in production, would give to the miller important information to adjust in the best way the process parameters (i.e. malaxation time, malaxation temperature, etc.) of the extraction plant or to choose between different machine alternatives or a combination of them. This would allow an improvement in process control. To achieve this aim, the use of alternative analysis technologies of the olive oil, such as electronic nose, could be a good opportunity (Aparicio et al., 2000; Esposto et al., 2006; Tena et al., 2007). To date there is only one research (Esposto et al., 2009) in which the monitoring of virgin olive oil volatile compounds during an industrial process was made by using an on-line electronic nose. The authors demonstrated the capability of the instrument to detect the formation and evolution of volatile compounds that characterize the most important sensory notes of VOO.

Recently, an innovative sensor BIONOTE (BIOsensor-based multisensorial system for mimicking NOse, Tongue and Eyes) has been developed (Santonico et al., 2013). The BIONOTE system (non-commercial prototype), which embeds gas and liquid sensors having a common biologically-derived sensing interface, allows the simultaneous analysis of the vapor and liquid phase of the samples and has been already used for VOO analysis to predict the adulteration of extra virgin olive oil with other vegetable oils analysing total polyphenols content, free acidity, peroxide value, and trolox equivalent antioxidant capacity (TEAC). The research demonstrated the ability of the electrochemical sensor to evaluate the presence of adulterants, proving to be a rapid tool for preliminary screening of the chemical characterization of the olive oil, aimed to detect samples that require further verifications by using traditional laboratory analysis.

In order to investigate further potential uses of BIONOTE the aim of this research was to evaluate the ability of the electrochemical sensor to analyse the headspace of olive oil and to find a correlation between the concentration of specific phenolic and volatile compounds and sensors responses, and also to evaluate the ability of the sensor to discriminate the olive oils samples originated by different technologies or by using different process parameters. The similarities and differences between the olive oil samples have also been highlighted.

\section{Materials and methods}

\section{Experimental design to obtain the olive oil samples}

The olive oil samples used in input to the electronic sensor were obtained by carrying out olive oil extraction cycles in AGROLIO s.r.l. mill, located in Andria (BT) in the south of Italy. The olive oil extraction plant was the model Cultivar 750 (MORI-TEM s.r.l., Tavernelle Val di Pesa, FI, Italy) which did not require the use of a vertical centrifuge for the separation of liquids. The industrial plant had a nominal capacity of $750 \mathrm{~kg} \mathrm{~h}^{-1}$.

Microwave and megasound prototypes units, developed by the authors and validated in previous research (Leone et al., 2017; Leone et al., 2018), were connected at the basic plant in order to obtain four different plant configurations explained below.

The tests have been carried out using olive fruits of cultivar Coratina (Olea europaea L.), having maturity index of 1.5 determined according to the method proposed by the International Olive Council (Uceda et al., 1975). Four different technologies to condition the olive paste (Figure 1) were used combining the traditional malaxer with microwave and megasound prototypes and described below: i) (ML) olive paste conditioning by using the traditional malaxer. Malaxation time $=30 \mathrm{~min}$ and malaxation temperature $=$ $28^{\circ} \mathrm{C}$; ii) (ML $\left.+\mathrm{MS}\right)$ olive paste conditioning by using the tradi-

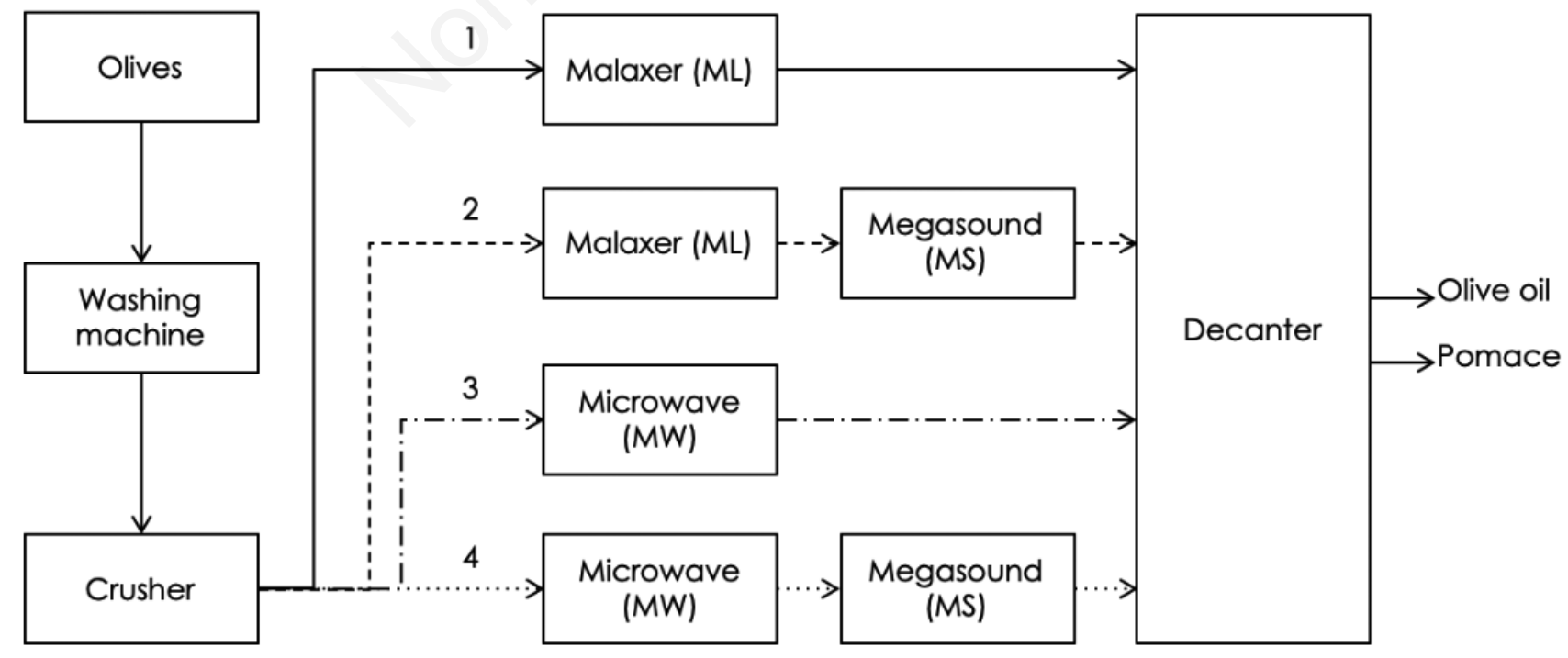

Figure 1. Path-lines of the four plant configurations. 
tional malaxer followed by the megasound prototype. Malaxation time $=30 \mathrm{~min}$, malaxation temperature $=28^{\circ} \mathrm{C}$; iii) $(\mathrm{MW})$ olive paste conditioning by using only the microwave prototype. Continuous microwave treatment for $55 \mathrm{~s}$ at constant temperature of $28^{\circ} \mathrm{C}$; iv) ( $\mathrm{MW}+\mathrm{MS}$ ) olive paste conditioning by using the microwave prototype followed by megasound prototype. Continuous microwave treatment for $55 \mathrm{~s}$ at constant temperature of $28^{\circ} \mathrm{C}$, followed by a continuous megasound treatment. Eight tests have been performed for each plant configuration. During each test two $100 \mathrm{ml}$ olive oil samples have been collected from the decanter at two different times (16 oil samples for each test and plant configuration). Every sample have been subsequently analysed five times with BIONOTE system.

\section{Chemical sensor}

BIONOTE is a multisensorial system based on different kinds of transducers: optical, gravimetric and voltammetric. In this work the authors have also implemented a sampling protocol optimized to analyse the sample headspace in liquid phase. In particular, the multisensorial system is based on a dedicated electronic interface in order to provide an optimized signal to noise ratio (SNR). The electronic circuit is composed of two blocks: one is configured to be wired with a probe while the other is a trans-impedance circuit used as I/V converter. The peculiarity of this system is that the output noise is very low and the spectral density of the equivalent current generator and the equivalent voltage generator are lower both

\section{$10^{-13} \mathrm{~A} / \sqrt{\mathrm{Hz}}$ and $10 \mathrm{nV} / \sqrt{\mathrm{Hz}}$.}

The voltammetric sensor employed in the liquid analyses consists of a sensing platform including a Screen-Printed Electrode [SPE; DRP-250BT, Metrohm Dropsens, S.L. (Llanera, Asturias, Spain)] probe (Working: Gold; Counter: Platinum; Reference: Silver), and a dedicated electronic interface providing the input signal and recording the output data. When the SPE is immersed in a solution, the applied input triangular waveform, from $+1 \mathrm{~V}$ to -1 $\mathrm{V}$, starts inducing oxidation-reduction phenomena to the analytes dissolved in the aqueous media. The current generated by the electrons involved in such reactions is converted in voltage by the trans-impedance circuit. The frequency of the input signal has been set to $0.01 \mathrm{~Hz}$ while the output signal was acquired with a rate of $200 \mathrm{~ms}$. This approach permits to obtain a reproducible pattern of current output values. The pattern is composed of 500 values considered as responses of 500 virtual sensors. Further details on the sensor and its application can be found in Santonico et al. (2013).

\section{Sensor measures of the olive oil samples}

After each olive oil extraction, a sample of olive oil $(100 \mathrm{~mL})$ has been sampled from the decanter (as reported above the extraction plant did not require the use of a vertical centrifuge for the separation of liquids) and subjected to the headspace measurement by BIONOTE sensor. Each measurement required to convey the olive oil headspace into an aqueous methanol solution $(50 \% \mathrm{v} / \mathrm{v})$ by means of a pneumatic system previously described by Pennazza et al. (2018). Briefly, the sampled olive oil was left within a hermetic dark glass container at $25^{\circ} \mathrm{C}$ for $30 \mathrm{~min}$ and then the generated headspace was forced to bubble into the solution for $180 \mathrm{sec}-$ onds allowing the volatile compounds to dissolve into the liquid media. The generated mixture was analysed immediately after the end of the bubbling step in order to minimize the release of the compounds back to the gas phase. The analyses with the multisensorial system were performed in real time at $25^{\circ} \mathrm{C}$ to reduce the possibility of olive oil sample oxidation through oxygen exposure or from light and temperature variations.

\section{Phenolic and volatile compounds analysis}

The analysis of olive oil phenolic and volatile compounds was performed in accordance with Servili et al. (2019).

\section{Statistical analysis}

Data analysis must be conducted using multivariate data analysis techniques: here a supervised method based on partial least square discriminant analysis (PLS-DA) is applied (Natale et al., 2006). Due to the multivariate nature of the data obtained during the production phase several prediction models were built having as input all the features extracted by the several approaches and as output a specific information about each analysed process. Data have been normalized to reduce the effect of systematic variation between samples by adjusting signal intensity by, for example: total intensity; highest value or intensity of a standard. The data set obtained from different approaches was treated considering the adjustment for sum, the mean or the standard deviation, respectively.In order to perform a hypothesis-driven (supervised) investigation, univariate analysis was explored, adjusted for multiple testing. For the fingerprint analysis an unsupervised technique (PCA) and a supervised technique (PLS-DA) were used. In particular, principal component analysis (PCA) is a statistical procedure that uses an orthogonal transformation to convert a set of observations of possibly correlated variables into a set of values of linearly uncorrelated variables, while PLS regression is a data analysis technique that combines characteristics from principal component analysis and multiple regression. In this context, it has been useful to predict a set of dependent variables from a large set of independent variables (predictors). To have a realistic error estimation, a cross validation technique was applied, retrieving RMSEC and RMSECV values. The technique used for the validation of the model is the leave one out: each of the measurements composing the data set is used as test-sample when it is left-out (leave-oneout) of the train set, formed by all the other measurements. Ratio performance deviation (RPD) value was calculated (Giovenzana et al., 2018) RPD is defined as the ratio between the standard deviation of the response variable and RMSECV. RPD values below 1.5 indicate that the calibration is not useful. When the RPD value is higher than 2, quantitative predictions are possible. Values in the range $1.5-2.0$, indicates that the algorithm has the possibility to distinguish between high and low values (Williams \& Norris, 2001). The best model calibrations were selected based on minimising the RMSECV and maximising RPD with a value greater or equal to 2.0. Multivariate data analysis was performed using PLS Toolbox (Eigenvector Research Inc., Manson, WA, USA) in Matlab Environment (The MathWorks, Natick, MA, USA).

\section{Results and discussion}

In the first step an unsupervised qualitative analysis has been performed to test the ability of the device to classify the four different plant configurations or technologies through the respective olive oil sample measurement. Figure 2 represents a bi-plot from the PCA analysis performed using values obtained by the sensor. Two first principal components explain $78.39 \%$ of total variance. The plot shows clusters corresponding to the different technologies, where technology ML (label 1), technology ML+MS (label 2), technology MW (label 3), technology MW+MS (label 4) and blank (methanol 70\% and water 30\%) (label 0), measurements used as reference, can be distinguished. Discrimination of different clusters along the two principal components highlights that the pat- 
terns associated to the sensor responses for each technology are different.

In Figure 3 an example of the BIONOTE pattern responses is reported. A first PLS-DA model was built in order to discriminate samples processed with the four different technologies. Table 1 shows the confusion matrix for the discrimination of the four technologies used in the processing.

To better define the ability of the BIONOTE system to identify the differences generated by the four technologies, the results of analyses of oil samples were grouped in three different ways to address the following hypotheses: i) the samples can be discriminated by the time of treatment. A: $\mathrm{t}<1$ '; B: $\mathrm{t}>1$ '; ii) the samples can be discriminated by the use of microwaves or not. A: MW used; B: MW not used; iii) the samples can be discriminated by the use of megasounds or not. A: MS used; B: MS not used.

The PLS-DA was then applied with the aim of identifying the treatment (A or B) relative to each hypothesis. All of them provided significant results. The first hypothesis was correct in the $98.44 \%$ of the cases (confusion matrix reported in Table 2) and this means that the olive paste conditioning time have significant effects on oil produced. In particular, the hypothesis A is relative to the microwave treatment (55"), and the B is relative to the other having a duration time of at least 30').

The second hypothesis was correct in the $100 \%$ of the cases

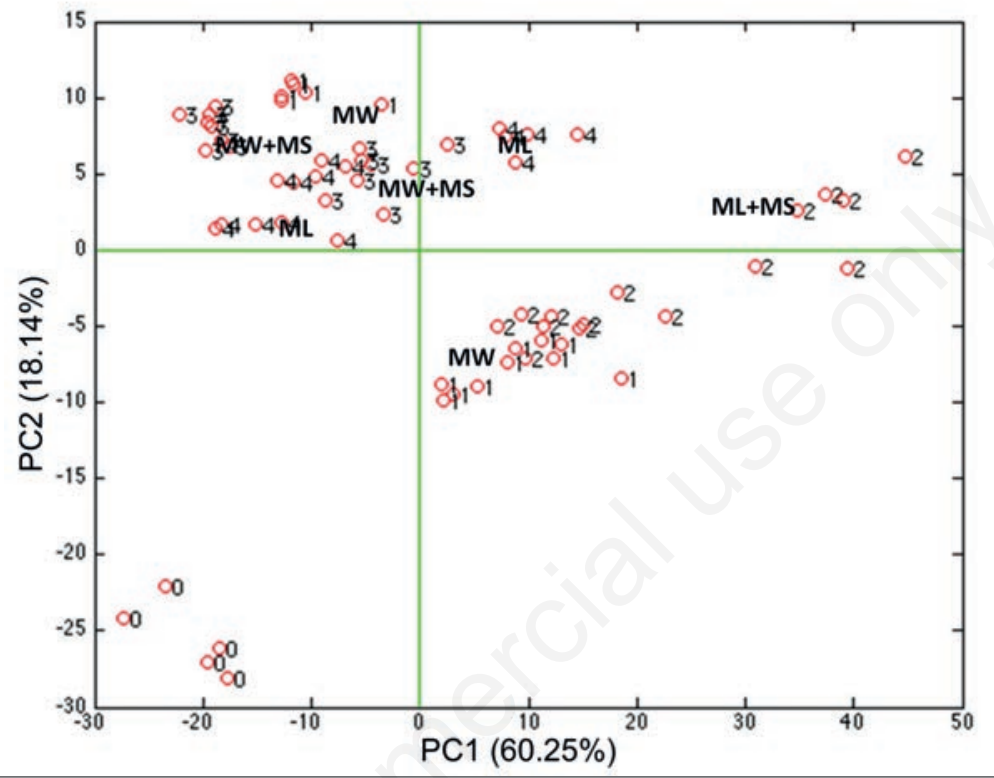

Figure 2. Scores plot of the first two principal components of the PCA model calculated on the data set of oil samples obtained by the four technologies used. Traditional malaxation ML (label 1), traditional malaxation and megasound technology ML+MS (label 2), microwave technology MW (label 3), microwave and megasound technology MW+MS (label 4) and blank (methanol $70 \%$ and water 30\%) (label 0).
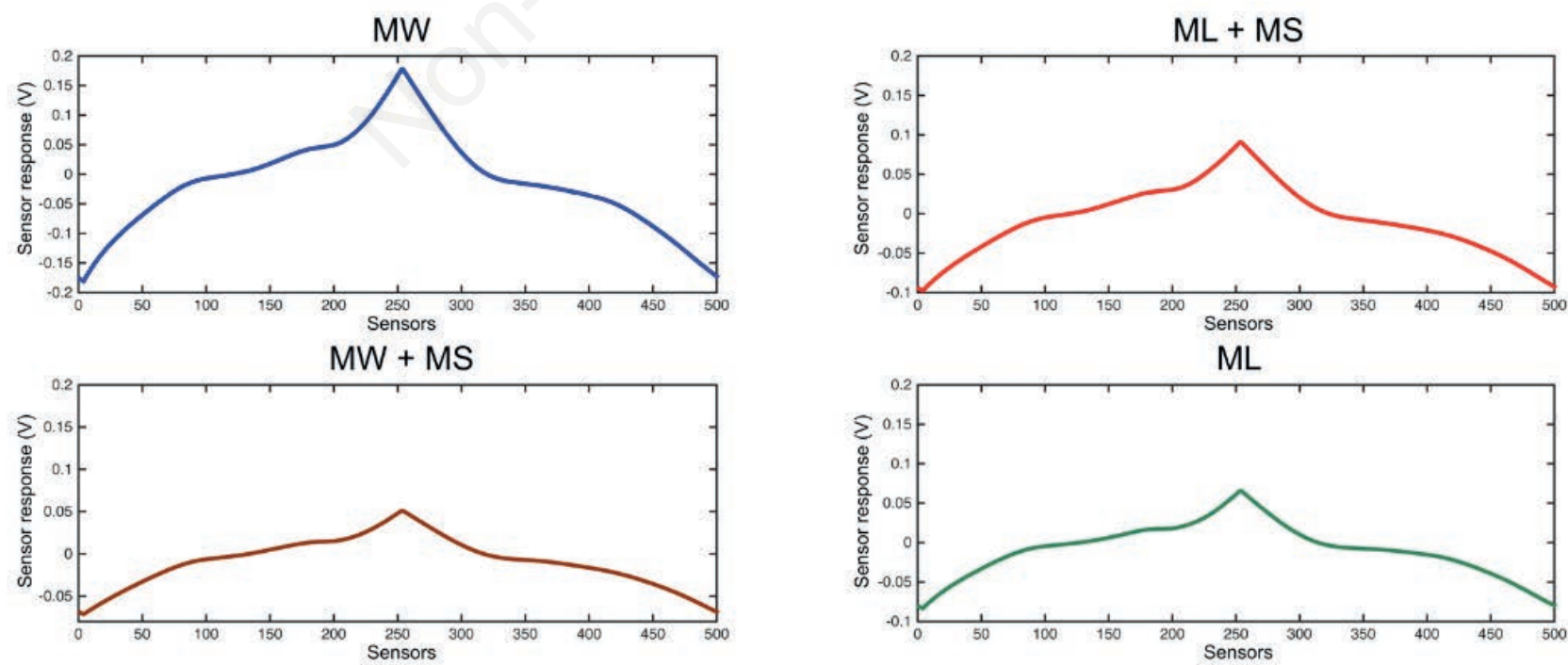

Figure 3. Response of the device for different samples. In particular, each graph reports the sensor voltage response (Volt) Vs the voltage applied input. These inputs are named sensors because they correspond to the 500 points of a periodic triangular wave between [-1, 1$]$ volt applied to the system. 
Table 1. Confusion matrix of the PLS-DA model for the discrimination of the four technologies used in the processing olive oil samples.

\begin{tabular}{lccccc} 
Technologies & \multicolumn{2}{c}{ Predicted technologies } & & Percentage of correct classification \\
& ML & ML + NS & MW & MW + MS & 100 \\
ML & 16 & 0 & 0 & 0 & 100 \\
ML + MS & 0 & 16 & 0 & 0 & 100 \\
\hline MW & 0 & 0 & 16 & 0 & 100 \\
MW + MS & 0 & 0 & 0 & 16 & 100 \\
Total & & & & &
\end{tabular}

Accuracy $=98.44 \% ;$ Sensitivity $=100 \% ;$ Specificity $=97.96 \%$.

(confusion matrix reported in Table 3). This result shows the characterization capability of MW technology on the oils produced. The sensors distinguished all the microwaves treated samples from those treated differently.

The third hypothesis was correct in the $92.19 \%$ of the cases (confusion matrix reported in Table 4), indicating that the BIONOTE system detected accurately the effect of megasounds on oil samples.

In the second step a correlation analysis has been performed to test the ability of the device to predict the concentration of the phenolic and volatile compounds in olive oil samples.

A PLS-DA model has been elaborated for each of the chemical compound listed in Table 5. In Table 6 the descriptive statistics PLS model statistics have been reported. Only the best correlated compounds have been reported (all the other compounds here not reported have shown a not-notable correlation value).

As shown in Table 6 the correlation is very high for 10 compounds, 4 phenolic compounds and 6 volatile compounds.

Considering the 4 phenolic compounds good predictive model were calculated for 3 compounds, considered important for healthprotective (Veneziani et al 2018), as secoiridoid derivatives of oleuropein (hydroxytyrosol (3,4-DHPEA)), ligstroside (tyrosol ( $p$ HPEA) and ligstroside aglycon) and for vanilic acid (RPD values ranged 2.01-2.14).

Good predictive results were also obtained for 6 volatile compounds (RPD values ranged 2.00 - 2.74), including 4 volatile compounds involved in the expression of positive sensory note of extra virgin olive oil released by the activation of lipoxygenase pathways: aldehydes $[(E)$-2-pentenal, hexanal, and (E)-2-hexenal), alcohol ((E)-2-penten-1-ol] (Genovese et al., 2015, 2018; Veneziani et al., 2018). On the basis of the importance of these compounds, their prediction in easy and simply way, in dependence of the process parameters, can have a good strategic role in the VOO processing management.

Results show that BIONOTE can be a good tool not only to
Table 2. Confusion matrix of the PLS-DA model for the discrimination of the two treatments (A or B) according to the first hypothesis (The samples can be discriminated by the time of treatment. A: $t<1$ '; B: $\left.\mathbf{t}>\mathbf{1}^{\prime}\right)$.

\begin{tabular}{lccc} 
Treatment & \multicolumn{2}{c}{$\begin{array}{c}\text { Predicted } \\
\text { treatment }\end{array}$} & $\begin{array}{c}\text { Percentage of correct } \\
\text { classification }\end{array}$ \\
& A & B & \\
A & 15 & 1 & 93.75 \\
B & 0 & 48 & 100.00 \\
\hline Total & & & 98.44 \\
\hline
\end{tabular}

Accuracy $=98.44 \%$; Sensitivity $=100.00 \%$; Specificity $=97.96 \%$.

Table 3. Confusion matrix of the PLS-DA model for the discrimination of the two treatments (A or B) according to the second hypothesis (The samples can be discriminated by the use of microwaves or not. A: MW used; B: MW not used).

\begin{tabular}{lccc} 
Treatment & \multicolumn{2}{c}{$\begin{array}{c}\text { Predicted } \\
\text { treatment }\end{array}$} & $\begin{array}{c}\text { Percentage of correct } \\
\text { classification }\end{array}$ \\
& A & B & 100.00 \\
A & 32 & 0 & 100.00 \\
B & 0 & 32 & 100.00 \\
\hline Total & & & 0 \\
\hline
\end{tabular}

Accuracy $=100.00 \%$; Sensitivity $=100.00 \%$; Specificity $=100.00 \%$.

Table 4. Confusion matrix of the PLS-DA model for the discrimination of the two treatments (A or B) according to the third hypothesis (The samples can be discriminated by the use of megasounds or not. A: MS used; B: MS not used).

\begin{tabular}{lccc} 
Treatment & \multicolumn{2}{c}{$\begin{array}{c}\text { Predicted } \\
\text { treatment }\end{array}$} & $\begin{array}{c}\text { Percentage of correct } \\
\text { classification }\end{array}$ \\
& A & B & \\
A & 27 & 5 & 84.38 \\
B & 0 & 32 & 100.00 \\
\hline Total & & & 92.19 \\
\hline
\end{tabular}

Accuracy $=100.00 \%$; Sensitivity $=100.00 \%$; Specificity $=100.00 \%$.

Table 5. Classes of the analysed chemical compounds.

\begin{tabular}{|c|c|c|c|c|}
\hline \multirow[t]{2}{*}{ Phenolic compounds } & \multicolumn{4}{|c|}{ Volatile compounds } \\
\hline & Aldehydes & Ketones & Alcohols & Esters \\
\hline 3,4-DHPEA & Pentanal & 3-pentanone & 1-pentanol & Hexyl acetate \\
\hline$p$-HPEA & (E)-2-pentenal & 1-penten-3-one & 1-penten-3-ol & (Z)-3-hexenyl acetate \\
\hline Vanilic acid & Hexanal & 6-methyl-5-hepten-2-one & (Z)-2-penten-1-ol & \\
\hline 3,4-DHPEA-EDA & (E)-2-hexenal & & (E)-2-penten-1-ol & \\
\hline$p$-HPEA-EDA & 2,4-hexadienal, (E,E) & & 1-hexanol & \\
\hline (+)-1-acetoxypinoresinol & 2,4-hexadienal, (i) & & (E)-2-hexen-1-ol & \\
\hline (+)-pinoresinol & & & (Z)-3-hexen-1-ol & \\
\hline 3,4-DHPEA-EA & & & Benzyl Alcohol & \\
\hline Ligstroside aglycone & & & Phenylethyl Alcohol & \\
\hline
\end{tabular}


Table 6. Descriptive statistics and PLS model statistics for the most correlated chemical compounds.

\begin{tabular}{|c|c|c|c|c|c|c|c|}
\hline Chemical compounds & $\mathrm{n}^{\circ}$ samples & Mean (mg/kg) & SD & LV & RMSE & RMSECV & RPD \\
\hline 3,4-DHPEA & 64 & 2.92 & 1.05 & 10 & 0.01 & 0.52 & 2.02 \\
\hline$p$-HPEA & 64 & 4.69 & 1.87 & 11 & 0.01 & 0.87 & 2.14 \\
\hline Vanilic acid & 64 & 0.69 & 0.22 & 11 & 0.00 & 0.10 & 2.13 \\
\hline Ligstroside aglycone & 64 & 26.08 & 5.31 & 6 & 0.98 & 2.50 & 2.12 \\
\hline (E)-2-pentenal & 64 & 112.97 & 24.31 & 11 & 0.03 & 12.17 & 2.00 \\
\hline Hexanal & 64 & 843.09 & 210.12 & 10 & 1.53 & 92.86 & 2.26 \\
\hline (E)-2-hexenal & 64 & 42984.69 & 13135.34 & 9 & 358.19 & 6466.90 & 2.03 \\
\hline (E)-2-penten-1-ol & 64 & 49.94 & 7.35 & 10 & 0.19 & 2.68 & 2.74 \\
\hline Benzyl Alcohol & 64 & 144.09 & 50.32 & 11 & 0.08 & 24.99 & 2.01 \\
\hline 6-methyl-5-hepten-2-one & 64 & 4.88 & 1.49 & 11 & 0.01 & 0.73 & 2.05 \\
\hline
\end{tabular}

SD, standard deviation; LV, latent variables; RMSE, root mean square error; RMSECV, root mean square error of cross validation; RPD, relative percent deviation.

detect chemical characteristics, such as free acidity, peroxidase index and TEAC and total polyphenols of olive oil, as reported by Santonico et al. (2015), but it can be useful to detect some phenols and volatile compounds with an high correlation value.

\section{Conclusions}

During the olive oil extraction process, knowing the progress of the formation of the phenolic and volatile components could be crucial to allow the miller to operate the adjustment of the process parameters or the choice between different technologies, providing that the measurement was rapid, inexpensive and technologically uncomplicated as anticipated by Esposto et al. (2009).

To this purpose BIONOTE system demonstrated its ability to discriminate different oil profiles, characterized by different processing technologies with different process parameter used. In addition, the correlation between some compounds analysed with laboratory analysis and the multisensor system used has been very high.

Furthermore, the PLS-DA models calculated shows that the system could provide an estimation of the olive oil compounds concentration. The results showed the system is able to follow modification of little entity in the sample (analysed at standard process temperature).

This study represents a first approach for the development of a simple and easy to use analysis tool. Anyway, to be able to make the device a rapid analyses tool in the mill, is necessary to increase the correlations between specific chemical compounds and the sensors by means of further investigations to assess the BIONOTE detection ability considering olive oil of different cultivars at different maturity indexes.

The future prospective is to insert this mathematical model in the microcontroller of the BIONOTE for a direct estimation of the olive oil chemical composition in the mill during the extraction process.

\section{References}

Angerosa F., Mostallino R., Basti C., Vito R. 2000. Virgin olive oil odour notes: Their relationships with volatile compounds from the lipoxygenase pathway and secoiridoid compounds. Food Chem. 3:283-7.
Angerosa F., Servili M., Selvaggini R., Taticchi A., Esposto S., Montedoro G.F. 2004. Volatile compounds in virgin olive oil: Occurrence and their relationship with the quality. J. Chromatogr. A 1054:17-31.

Aparicio R., Rocha S.M., Delgadillo I., Morales M.T. 2000. Detection of rancid defect in virgin olive oil by the electronic nose. J. Agr. Food Chem. 48:853-60.

Cavalli J.F., Fernandez X., Lizzani-Cuvelier L., Loiseau A.M. 2003. Comparison of static headspace, headspace solid phase microextraction, headspace sorptive extraction, and direct thermal desorption techniques on chemical composition of French olive oils. J. Agr. Food Chem. 51:7709-16.

Esposto S., Servili M., Selvaggini R., Riccò I., Taticchi A., Urbani S. 2006. Discrimination of virgin olive oil defects. Comparison of two evaluation methods: HS-SPME-GC/MS and electronic nose. In: M.A. Petersen \& W.L.P. Bredie (Eds.), Flavour science: Recent advances and trends. Developments in food science. Elsevier B.V. Publisher, Amsterdam, The Netherlands, 43:315-8.

Esposto S., Montedoro G.F., Selvaggini R., Riccò I., Taticchi A., Urbani S., Servili M. 2009. Monitoring of virgin olive oil volatile compounds evolution during olive malaxation by an array of metal oxide sensors. Food Chem. 113:345-50.

Flath R.A., Forrey, R.R., Guadagni D.G. 1973. Aroma components of olive oil. J. Agr. Food Chem.21:948-52.

Genovese A., Caporaso N., Villani V., Paduano A., Sacchi R. 2015. Olive oil phenolic compounds affect the release of aroma compounds. Food Chem. 181:284-94.

Genovese A., Yang N., Linforth R., Sacchi R., Fisk I. 2018. The role of phenolic compounds on olive oil aroma release. Food Res. Int. 112,:319-27.

Giovenzana V., Beghi R., Romaniello R., Tamborrino A., Guidetti R., Leone A. 2018. Use of visible and near infrared spectroscopy with a view to on-line evaluation of oil content during olive processing. Biosyst. Engine. 172:102-9.

Leone A., Romaniello R., Tamborrino A. 2013. Development of a prototype for extra-virgin olive oil storage with online control of injected nitrogen. Trans. ASABE 56:1017-24.

Leone A., Romaniello R., Zagaria R., Sabella E., De Bellis L., Tamborrino A. 2015a. Machining effects of different mechanical crushers on pit particle size and oil drop distribution in olive paste. Eur J. Lipid Sci. Tech. 117:1271-9.

Leone A., Romaniello R., Zagaria R., Tamborrino A. 2015 b. Mathematical modelling of the performance parameters of a 
new decanter centrifuge generation. J. Food Enginee. 166:10-20.

Leone A., Romaniello R., Tamborrino A., Xu X.-Q., Juliano P. 2017. Microwave and megasonics combined technology for a continuous olive oil process with enhanced extractability. Innov. Food Sci. Emerg. Technol. 42:56-63.

Leone A., Romaniello R., Tamborrino A., Urbani S., Servili M., Amarillo M., Grompone M.A., Gambaro A., Juliano P. 2018. Application of microwaves and megasound to olive paste in an industrial olive oil extraction plant: impact on virgin olive oil quality and composition. Eur. J. Lipid Sci. Tech. 120:1700261.

Natale C.D., Martinelli E., Pennazza G., Orsini A., Santonico M. 2006. Data analysis for chemical sensor arrays. pp 147-169 in Advances in Sensing with Security Applications, Springer: Dordrecht, The Netherlands.

Pennazza G., Santonico M., Zompanti A., Parente F.R., Ferri G., D'Amico A. 2018. Design and development of an electronic interface for gas detection and exhaled breath analysis in liquids. IEEE Sens. J. 18:31-6.

Salas J.J., Sánchez J. 1999. Hydropeoxyde lyase from olive (Olea europaea) fruit. Plant Sci. 143:19-26.

Santonico M., Pennazza G, Grasso S., D’Amico A., Bizzarri, M. 2013. Design and test of a biosensor-based multisensorial system. Sensors 13:16625-40.

Santonico M., Grasso S., Genova F., Zompanti A., Parente F.R., Pennazza G. 2015. Unmasking of olive oil adulteration via a multi-sensor platform. Sensors (Switzerland). 15:21660-72.
Servili M., Esposto S., Fabiani R., Urbani S., Taticchi A., Mariucci F., Montedoro G.F. 2009. Phenolic compounds in olive oil: Antioxidant, health and organoleptic activities according to their chemical structure. Inflammopharmacol. 17:76-84.

Servili M., Veneziani G., Taticchi A., Romaniello R., Tamborrino A., Leone A. 2019. Low-frequency, high-power ultrasound treatment at different pressures for olive paste: Effects on olive oil yield and quality. Ultrason. Sonochem. 59:104747.

Tamborrino A., Pati S., Romaniello R., Quinto M., Zagaria R., Leone A. 2014. Design and implementation of an automatically controlled malaxer pilot plant equipped with an in-line oxygen injection system into the olive paste. J. Food Eng. 141:1-12.

Tena N., Lazzez A., Aparicio-Ruiz R., García-González D.L. 2007. Volatile compounds characterizing Tunisian Chemlali and Chétoui virgin olive oils. J. Agr. Food Chem. 55:7852-8.

Uceda M., Frias L. 1975. Harvest dates: evaluation of the fruit oil content, oil composition and oil quality. pp 125-128 in Proc. Second Int. Meeting of Olives Oil, Cordoba, Portugal.

Veneziani G., Esposto S., Taticchi A., Urbani S., Selvaggini R., Sordini B., Servili M. 2018. Characterization of phenolic and volatile composition of extra virgin olive oil extracted from six Italian cultivars using a cooling treatment of olive paste. LWT - Food Science and Technol. 87:523-8.

Williams P., Norris K. 2001. Near infrared technology in the agriculture and food industries. Am. Assoc. Cereal Chem. Inc., St. Paul, MN, USA. 